2B several lethal complementation groups have been identified and some alleles seem to show abnormalities in the normal temporal sequence of gene activities inducible by ecdysone in salivary glands. Since the $2 B$ puff is one of the first to respond to the hormone the implications of these observations for the analysis of cascade control reactions during development are clear to see.

\section{Ecdysone receptors}

Another topic of enduring interest is that of the general control of development by ecdysone. The meeting was fascinated by accounts of two temperature-sensitive ecdysone-deficient mutants by J. Lepesent (University of Paris) and J. Holden (Queen's University, Kingston), which will be invaluable for the study of ecdysone action. Even more fundamental, however, is the problem of the ecdysone receptors. So elusive have these been that some were beginning to doubt their very existence on anything other than theoretical grounds. The breakthrough by J. D. O'Connor (University of California, Los Angeles) and by Fristrom's group in Berkeley, has now been made. What was needed was a radiolabelled analogue of ecdysone of very high biological activity and very high specific activity. This was synthesised by the tritiated reduction of the phytoecdysone stakisterone $\mathrm{C}$ to the very active ponasterone A. Using tissue culture cells O'Connor and his group have characterised a proteinaceous binding activity that does everything an ecdysone receptor should do, at least to those acquainted with vertebrate steroid receptors. Thus the binding shows a temperature-dependent nuclear translocation, a translocation accompanied by a change in sedimentation constant, it is saturable and shows biologically realistic affinity constants to various ecdysone analogues. Moreover derivatives of the original cell line exist which are unresponsive to the hormone and at least some of these seem to lack the receptor. The receptor's purification and localisation to ecdysone-treated salivary gland chromosomes is eagerly anticipated. If current attempts to isolate the ecdysone-responsive genes on

\section{Erratum}

IN the article 'Tllegitimate recombination legitimised' (News and Views 274,$213 ; 1978)$ the reference (column 3 , page 213 , line 10 ) to the 9-base pair repeat generated by the integration of insertion element IS4 and attributed to P. Starlinger, is incorrect. The repeated sequences generated by IS 4 have not yet been determined. plasmids succeed we are in for an exciting few years.

\section{Sex determination}

R. Nöthiger and his colleagues (University of Zurich) are throwing new light on an old problem, at least as far as Drosophila is concerned. For years Drosophila geneticists have accepted the balance theory of sex determination of Bridges, whereby sex is determined by the ratio between the number of $X$ chromosomes and autosomes, without enquiring too deeply just what this may mean in genetic or developmental terms. Nöthiger suggests that, all organisms, whether man, beast, or green things, possess a 'key gene' whose activation or inactivation is required for sexual development along one or other pathway. In different organisms different methods will be used to control the activity of this key gene: in some (the marine worm Bonellia) the signal will be environmental, in others genetic. In Drosophila it would be this key gene that responds, by an unknown mechanism, to the $\mathbf{X}$ chromosome : autosome balance. The report of $T$. Cline of Princeton University of a new locus on the $\mathrm{X}$ chromosome, which appears to be normally 'on' in females and 'off' in males, and may thus correspond to Nöthinger's proposed key gene, is therefore particularly exciting. Cline has found that if the normal state of affairs is disturbed by mutation, sex-limited lethality results. Nöthiger's laboratorv has been systematically analysing the several mutant loci known in Drosophila which effect a sex reversal and it was suggested that perhaps the activity of their wildtype alleles is normallv subordinate to that of the X-linked key gene.

\section{Cell division and death}

The control of cell division and cell dcath in growing organs remains a mystery, but Drosophila geneticists are making a start on its analysis. It has been known for some time that when wild-tvne cells are generated in a slow growing (Minute/Minute ${ }^{+}$) background they grow fast and take over much of the compartment. Morata and Ripoll (Devl. Biol. 42, 211; 1975) discovered that when the converse experiment is done-slow growing clones generated in a wild-tyne background-the weaker cells are completely eliminated. Yet comvartments (or indeed whole flies) consisting entirely of Minute cells form normally (Ferrus \& Garcia-Bellido, Wilhem-Roux Arch. 183, 337; 1977). This phenomenon. termed cell competition by its discoverers, was discussed by P. Simpson (Gif-sur-Yvette) who suggested that the relative growth rate of the cells is the critical factor. Minute cells generated in a Minute background, for example, survive. Further, starvation which reduces the total amount of growth, diminishes the effects of competition, rescuing the weaker Minute cells. It seems that some kind of cellular struggle which results in survival of the fittest is operating in growing cell populations.

J. Szabad (Szeged) reported results obtained in collaboration with $T$. Schüpbach and E. Wieschaus (University of Zurich). They have made gynandromorphs of Drosophila larvae and studied the distribution of male and female epidermal cells. These gynandromorphs are in many respccts more valuable than adult ones, as they are a more direct representation of the blastoderm. Thanks to these studies we now know that the $s /$ o boundary line on the blastoderm is convoluted, and that segmentation coincides with blastoderm stage. For a long time the relationship between the cells on the blastoderm and the gynandromorph fate map has remained uncertain. We now know that each cell on the blastoderm is represented by about 4 sturts on the fate map.

It is always debatable whether conferences are really worth it. In the case of Szeged there can be no question that the formal discussions, and more importantly, the informal ones (especially between colleagues who, for the wrong reasons, so rarely meet) were very worth while.

\section{The LEP \\ summer study}

from a Correspondent

Much progress in understanding subnuclear constituents has been made using a new facility for probing the structure of matter at extremely small distances $\left(\sim 10^{-14} \mathrm{~cm}\right)$, namely the electron-positron storage ring. In such a machine beams of electrons and positrons collide head-on producing a single massive photon. which can then pair produce other, heavier particles. This process is quite democratic; any charged particle which exists with a mass less than the equivalent energy of one of the electron beams must be produced with a calculable cross section. Furthermore the production process is 'clean'. The pair of new particles is made alone, devoid of the debris of other subnuclear particles which is always present if the proton beam of a conventional accelerator and a fixed target is used.

The basic constituents of matter seem to be fermions, occurring in families of four, each containing two quarks and two leptons. These fermions 